Lymphoedema (lymphoedema), which is understood to be a result of mechanical (low output) failure of the lymphatic system, may be a result of abnormalities of structure and function of the lymphatic system (primary lymphoedema), injuries, inflammation, chronic vein insufficiency or related to cancer (secondary lymphoedema).

Because of the chronic nature of the illness and the lack of possibilities to be cured, education and prevention play an important role.

The purpose of this paper is to present a current review of using prophylactic methods in the prevention of lymphatic insufficiency.

Key words: lymphatic system, lymphedema, lymphoedema, prevention, education, physical activity, compression.

\section{Prevention of lymphoedema}

\author{
Katarzyna Ochałek
}

University School of Physical Education in Cracow Lymphoedema Clinic, St Lazarus Hospice in Cracow

There is no doubt that it is easier to prevent diseases than to treat them. Prophylaxis (from the Greek word phylax - take care, prohylaktikos - preventive), even though it is not a domain of medicine, is related to all aspects of life; it means activities whose aim is to prevent disease development (early prevention), control causes and risk factors related to people at risk (primary prophylaxis) or decrease its results (secondary prophylaxis), in other words maintenance of the status quo of the organism.

At every stage of preventive activities, health education related to the form of definite attitudes and behaviours with inquiry activities and realization of the relationship between health and lifestyle play a significant role. These activities, which include physical effort, way of nutrition, avoiding overweight, coping with stress and use of stimulants in connection with physical treatment, can also efficiently prevent lymphoedema occurrence.

\section{Aetiopathogenesis of lymphoedema}

Lymphoedema, regarded as a result of mechanical insufficiency (low output failure), resulting in decreased transport capacity, may be a result of abnormalities of structure and function of the lymphatic vessels (primary lymphoedema), injuries, inflammation or related to cancer (secondary lymphoedema).

Primary lymphoedema is a result of rare teratogenic defects of the lymphatic system that may occur in children as an early or in adults as a delayed form of lymphoedema [1].

Lymphoedema in advanced cancer may occur in consequence of the occlusion or compression of lymph vessels by the disease process, in people without advanced cancer, after surgery or radiotherapy which included lymph nodes; there is sometimes latent, subclinical lymphatic insufficiency [2].

In chronic vein insufficiency, as a result of increase of hydrostatic pressure in the vein vessels, a lot of the fluid formed cannot be transported to the circulation by the lymphatic system, which in time causes an impairment of the valve system and in consequence the whole lymphatic system [3].

\section{Clinical picture}

Lymphoedema is usually asymmetrical; it affects mostly one limb, sometimes close-fitting parts of the trunk. At first it is not large; untreated over time it becomes hard. The volume of the limb increases, limited range of motion and muscle contractures appears.

These changes lead to susceptibility of the skin to injuries and damage as a result of which occur lymphorrhoea and secondary infection (cellulitis, erysipelas), in extreme cases to development of cancer such as lymphosarcoma, epithelial carcinoma, lymphoma or melanoma.

Aside from of the reason lymphoedema is a chronic disease without possibilities to be cured, limits physical fitness and causes problems of a psychosocial nature decreasing quality of life [4].

\section{Therapeutic management}

From these important for the life and the health results, prevention, patient education and their family play an essential role in the area of acts pre- 
venting to the pronouncement of this complication. In every patient who has experienced surgical, orthopaedic, or oncological treatment, there grows the risk of lymphoedema development; simple preventive methods ought to be introduced, but in case of its development, treatment should begin quickly. Prophylaxis together with education are the most important part of lymphoedema care [5].

Current studies show lack of awareness and a low level of education of people threatened with swelling, and difficulties with access to the professional literature on the subject of prophylaxes of this illness. A source of knowledge on the subject related to lymphoedema is seldom properly qualified medical staff [6].

Among factors inducing this complication most often one mentions injuries, lack of physical activity or excessive physical effort, overwork, heat and overweight [7]. The elimination of the above-mentioned factors, and the knowledge of essential and preventive acts reduce the risk of development of lymphoedema and its results. These acts include skin and nail care, avoiding exposure to different kinds of injury, appropriate physical exercises, MLD usage, maintenance of proper body weight, elevation of the limb and use of compression in some cases.

\section{Skin and nail care}

The proper skin care and nails targets the maintenance their fit so the exclusion to the infection (inflammatory, mycotic), which can cause the development of lymphoedema. Appropriate hygiene of the skin can be obtained by washing every day with hypoallergenic soap, exact drainage, especially places between fingers (toes) and moisturizing by application proper means (balms, creams, the olive-tree). One ought to try to protect the skin before all types of injury: mechanical (injections, blood pressure measurement, blood test, live-stock scratch, hurts, insect bites, tight-fitting jewellery), chemical (contact with irritating detergents), thermal (heat, cold, sun exposure). In case of the development of the infection of the skin in the area of subject on the risk of the pronouncement of the swelling, a visit to the doctor, with the aim of the use of an antibiotic, is indispensable [8].

\section{Physical activity}

Regular, intensive physical activity in the form of physical and breathing exercises performed within an adequate period of time is considered to be an integral part of the prophylaxis of lymphatic insufficiency.

It is proper to underline that both a lack of physical activity as well as too vigorous and prolonged sport activity are not beneficial. Without movement and exercises, lymph flow falls to a very low level; high-intensity or prolonged physical activity increases local congestion which leads to overproduction of the fluid and can develop or increase lymphoedema.

Physical activity should include exercises maintaining or improving the range of motion in the shoulder, strengthening muscles, generally increasing fitness and maintenance of body weight. An optimal exercise programme should include exercises activating the mechanism of the muscle pump, exercises based on dynamic physical efforts: active, alleviat- ed and self-supported exercises, swimming, biking, walking, jogging. It is advised to do physical activity at a moderate intensity with a small number of repetitions, several times during a day so not to tire muscles. High-intensity and/or prolonged physical effort, especially without appropriate preparation, may lead to developing lymphoedema. It is advised also to avoid isometric exercises which rely on a change of muscle tension without a motor effect (carrying shopping, driving), anaerobic physical efforts (resistive exercises, weight lifting, short distance running, some asanas in yoga), which increase lymph flow and in consequence can induce or intensify lymphoedema [9].

\section{Manual lymph drainage/Simple lymph drainage}

Based on the scientific reports, traditionally a simplified version of manual lymph drainage (MLD) is advised - simple lymph drainage (SLD) [10]; however, the latest observations evidence the efficiency only of manual lymph drainage. The study comparing MLD usage in connection with physical exercises and an educational programme in the early period after mastectomy proves the rarer occurrence of lymphoedema compared to the group which only took an educational programme [11]. The aim of manual lymph drainage, which influences mainly superficial parts of the lymphatic system, in people at risk, is improving lymph flow in non-fibrotic lymph vessels. It starts from proximal, unaffected part of the body (the neck, chest, abdomen) and after preparation of the healthy part of the lymphatic system, the threatened regions are treated (usually upper or lower limb) starting proximally and moving progressively down the limb with such pressure not to cause skin reddening or pain. Otherwise excessive high pressure influences tissues and can damage the lymph vessels. Based on the simple techniques and rules of MLD, one worked out his simplified version performed by patients or trained members of families.

\section{Compression}

Despite the method with the use of the compression so of the pressure is not evaluated univocally as effective in the prophylaxis of lymphoedema, in selected cases, for example in people performing heavier physical work, practising sport, in travellers or in immobilized people, remaining in a compulsory position for several hours, especially after gynaecological operation [12], it can be advised to use compression garments: arm sleeves, stockings or knee-stockings [3]. It is the simplest type of compression which generates graduated pressure and maintains constant pressure.

Compression garments differ in the degree of pressure (compression class) and elasticity; there are different indications for their use and for these reasons they should be chosen individually in respect of the size and the compression class by experienced physiotherapists or nurses.

\section{Maintenance of proper body weight}

Maintenance of the correct mass of the body (body mass index, $\mathrm{BMI} \leq 25 \mathrm{~kg} / \mathrm{m}^{2}$ ) is a preventive and curative factor of lymphoedema. There is a relationship between the occurrence of overweight or obesity (BMI $\left.>25 \mathrm{~kg} / \mathrm{m}^{2}\right)$ and the development of lymphoedema [13]. 
For the purpose of the maintenance of the correct mass of the body one should follow a varied diet: low-salt, rich in vitamins (fruit and vegetables), richly residue (dark bread, cereals) and limitation of stimulants (alcohol, strong coffee, tea).

\section{Elevation of the limb}

Elevation of the limb to heart level several times during a day for about 10-15 minutes is essential; at night it recommends the use of a pillow or special wedges (accessible with the medical equipment) making higher elevation of the limb easy.

The aim is to use gravitational forces for improvement of the lymph outflow.

Lymphoedema due to oncological treatment significantly decreases quality of life. Education with the knowledge of rules of prophylaxis and early physiotherapy allow one to decrease the risk of lymphoedema and its complications. The propagation of the knowledge related to prevention of lymphoedema, and initiation of the simple preventive method, is a task which should be realized in progress or just after the end of treatment in oncological centres and rehabilitative clinics.

Valuable help can also be provided by organizations, associations (e.g. "Amazones"), foundations (e.g. Polish Foundation of Lymphology), signalling the scale of the problem contributing to the wider spread of knowledge related to efficient methods of prevention and the possibility of treatment.

In case of developing lymphoedema and/or its complications, not as usual important is clinical assessment and quick initiation of treatment based on physical therapy including compression therapy (compression bandaging, intermittent pump compression, fitting of compression garments) in connection with manual lymph drainage and physical exercises [14, 15].

The system of guaranteed services in lymphoedema treatment demands modification. In the present system patients using interventions, in spite of the improvement of short duration, are not included in prolonged therapeutic programmes and in case of lymphoedema progression or its complications they often cannot urgently begin intensive treatment.

Additionally, only selected procedures (manual lymph drainage and physical exercises) are contracted by the National Health Service in the range of systemic rehabilitation and remaining elements of the treatment which enters into \{composition\} of the lymphoedema therapy, in other words compression therapy (compression bandaging, compression garments) do not exist in financed procedures in health service. For these reasons it pays attention to the extension of guaranteed services for the physiotherapy of lymphoedema in the range of the day's rehabilitation and it reports the urgent need of the elaboration of the standard-model in the management of this illness.

\section{References}

1. Damstra RJ, Mortimer PS. Diagnosis and therapy in children with lymphedema. Phlebology 2008; 23: 276-86.

2. Hayes SC, Janda M, Cornish B, Battistutta D, Newman B. Lymphedema after breast cancer: incidence, risk factor and effect on upper body function. J Clin Oncol 2008; 21: 3536-42.
3. Partsch H, Flour M, Coleridge-Smith PD. Consensus statement. Indications for compression therapy in venous and and lymphatic disorders. Int Angiol 2008; 27: 193-219.

4. Ahmed RL, Prizment A, Lazovich D, Schmitz KH, Folsom AR. Lymphedema and quality of life in breast cancer survivors: the lowa Women's Health Study. J Clin Oncol 2008; 10: 5689-96.

5. Boccardo F, Michelini S, Eretta C, et al. Prevention of Lymphedema surgery for breast cancer. The European Journal of Lymphology and Related Problems 2007; 17: 24-8.

6. Ridner SH. Pretreatment lymphedema education and identified educational resources in breast cancer patients. Patient Educ Couns 2006; 1: 72-9.

7. Park J, Lee W, Chung $\mathrm{H}$. Incidence and risk of breast cancer lymphoedema. J Clin Nurs 2008; 17: 1450-9.

8. Vignes S, Dupuy A. Recurrence of lymphoedema - associated cellulites (erysipelas) under prophylactic antibiotherapy: a retrospective cohort study. J Eur Acad Dermatol Venereol 2006; 20: 818-22.

9. Ochałek K. Physical activity in the prevention and treatment of lymphatic insufficiency. Medicina Sportiva 2008; 4: 146-9.

10. Woods M. The experience of manual lymph drainage as an aspect of treatment for lymphedema. Int J Palliat Nurs 2003; 8: 336-41.

11. Torres Lacomba M, Yuste Sánchez MJ, Zapico Goñi A, Prieto Merino D, Mayoral del Moral O, Cerezo Téllez E, Minayo Mogollón E. Effectiveness of early physiotherapy to prevent lymphoedema after surgery for breast cancer: randomized, single blinded, clinical trial. BMJ 2010 340: b5396 (on line).

12. Casley-Smith JR. Lymphedema initiated by aircraft flights. Aviat Space Environ Med 1996; 67: 52-6.

13. Shaw C, Mortimer P, Judd P. A randomized controlled trial of weight reduction as a treatment for breast cancer-related lymphedema. Cancer 2007; 8: 1868-74.

14. International Society of Lymphology. The diagnosis and treatment of peripheral lymphedema. 2009 Concensus Document of the International Society of Lymphology. Lymphology 2009; 42: 51-60.

15. Ochałek K, Gradalski T. Manual lymph drainage may not be a necessary component in lymphedema treatment. J Pain Symptom Manage 2010; 5: 1-2

\section{Address for correspondence}

\section{Katarzyna Ochałek}

Lymphoedema Clinic

St Lazarus Hospice in Cracow

Fatimska 17

31-831 Cracow 